\title{
CHEMICAL INVESTIGATION OF MEDICAL MUD FROM LAKE NOGOON
}

\author{
Dolmaa G. ${ }^{1}$, Bayaraa B. ${ }^{1 *}$, Tserenkhand B. ${ }^{2}$, Nomintsetseg B. ${ }^{1}$, Ganzaya G. ${ }^{1}$ \\ ${ }^{1}$ Institute of Chemistry and Chemical Technology, Mongolian Academy of Sciences, Mongolia \\ ${ }^{2}$ School of Chemistry and Chemical Engineering, National University of Mongolia, Mongolia \\ *corresponding author, e-mail: bayaraabatnasan@gmail.com
}

ARTICLE INFO: Received: 26 Sep, 2017; Revised: 15 Dec, 2017; Accepted: 20 Dec, 2017

\begin{abstract}
The purpose of study was to determine chemical composition and organic matter of peloid from Nogoon Lake in Govi-Altai province by chemical and several analytical techniques. Based on these studies, it was identified that peloid belongs to silt sulphide muddy type, which is included the polymineral group. The content of macro and microelements in peloid from Nogoon was $0.0003-42.65 \%$. Free organic matters in peloid were extracted using non-polar and polar solvents. Yield of total extract in peloid from Lake Nogoon was $1.479 \%$.

We determined chloroform-dissoluble organic matter (DOM) in total extract's dry residue after consistently extracted by organic solvents. There were used chromato-mass-spectrometric method and identified 83 organic compounds in peloid from Lake Nogoon, that were containing hydrocarbons derivatives $33.68 \%$, carboxylic acid $12.95 \%$, dialkylphatalate (DAF) $3.09 \%$, ketone $1.01 \%$ and nitrogen-containing compound $3.96 \%$. The IR spectrum of peloid from Lake Nogoon showed CH2, CH3 alkanes group, carboxylic acid, ether (C-O) group, alkanes (CH) group absorption that free organic matter in peloid consist multiple compounds. We have developed a route for isolating and determining the composition of the organic compounds in peloids.

Lipid group contain fat-soluble vitamins include vitamins $A, D, E$ and their derivatives, steroids and other biological activity compounds which are similar to lipid by chemical and physical characterization. These compounds have shown lot of action such as oxidation and inflammation, microbial and membrane stabilization. Furthermore, the lipid fraction of the medical mud is widely used in cosmetics [1]. In the peloid from Lake Nogoon lipid matter contained 55 organic compounds alkanes 47.73\%, DAF 5.58\%, carboxylic acid $0.7 \%$, ester $0.3 \%$, steroids $17.24 \%$, oxygen organic compound $0.6 \%$, and element sulfur $2.88 \%$, nitrogen-containing compound $2.33 \%$, silicon-containing compound $3.74 \%$, cyclic hydrocarbons $0.27 \%$ respectively.
\end{abstract}

Keywords: peloid; silt sulphide mud; humic matter; lipid; carbohydrate;

\section{INTRODUCTION}

Peloids are natural great product and multi-component system, which consists of minerals, water, salt, free and linked organic matter and organo-mineral complex, micro and macro elements, macromolecular compounds. Peloids formed a very long period of time by chemical, physical, biological and geological processes, and that became healthfulness [2]. The first recorded use of medical mud goes back to ancient Greekbut research work of medical mud was not performed until $19^{\text {th }}$ century. Nowadays, many country studied chemical composition and properties of own country's peloid. For example, Russian 
scientists carried out extensive research on the chemical nature and therapeutic properties of the sapropel and peat (Bakhman and Ovsyanikova, 1965; Puntus, 1998, 2008; Shinkarenko and Milenina, 1981; Shustov, 1996). There are more than hundred lakes with medical mud in Mongolian but we have studied physicochemical properties and some

\section{MATERIAL AND METHODS}

\section{Sampling area}

Lake Nogoon in Gobi-Altai province: Lake Nogoon located $12 \mathrm{~km}$ from Gobi-Altai city. People in Gobi-Altai aimag named it stinky Lake instead of Nogoon Lake because the lake has a stinky smell [3]. The geographic coordinates of Lake Nogoon are $45^{\circ} 22^{\prime} 59^{\prime \prime} \mathrm{N}$, $96^{\circ} 15^{\prime} 48.5^{\prime \prime} \mathrm{E}$.

Methods: We used MNS 5848:2008 standard for taking and preparing samples [4]. General characteristics of peloids determined by MNS 5849:2008 [5] standard, mineral composition in peloid by X-Ray diffractometer (Rigaku, MAXimax) and chemical composition of peloid by roentgen fluorescence energy disperse MESA 500W. Yield of lipid and humic substances determined by MNS5849:2008 and MNS 5442:2005 standards $[6,7]$, respectively.

\section{RESULTS AND DISCUSSION}

\section{General characterization of peloid from Lake Nogoon}

At first general characterization of peloid was performed, because itdetermines their classification. For the investigation of general organic compound of medical mud, which is collected from 45 lakes. The main objective of this paper is to determine the chemical, physical composition of natural medical mud from Nogoon Lake and to provide some fundamental data which are useful to explain the therapeutic effects of peloid.

The yield of free organic matter in peloid was determined with the following conditions: It was washed by distilled water with the purpose of cleaning from salts, dried and sequentially extracted with hexane, chloroform, acetone, and mixture of ethanol: benzene (1:9) using Soxhlet apparat. The compositions of organic compounds were determined by chromatomass-spectral method and infrared spectroscopy. The mass-spectra recorded on chromatomass-spectrometer LKB-2091 (HP 5971A), by using of capillary columns with phases BD 5, OV 70-280, SE54 , SE-30, length 25 and $60 \mathrm{~m}$ in automatically programmed temperature mode, the speed of temperature expansion was 4 grade/minute. IR spectra were recorded on spectrometer IR-20 using $\mathrm{KBr}$ pellets.

Table 1. General characteristics of peloid from Lake Nogoon compared With the international classification of peloid

\begin{tabular}{|c|c|c|c|c|c|}
\hline \multirow{2}{*}{$\begin{array}{c}\text { General } \\
\text { characteristics }\end{array}$} & Peat & Sapropelic & Silt sulphide & Knoll & $\begin{array}{c}\text { Peloid from } \\
\text { Lake Nogoon }\end{array}$ \\
\cline { 2 - 5 } Color & $\begin{array}{c}\text { brown, } \\
\text { dark brown, } \\
\text { black }\end{array}$ & $\begin{array}{c}\text { gray, } \\
\text { greenish, } \\
\text { dark brown }\end{array}$ & dark gray & gray & dark gray \\
\hline Moisture, \% & $60-80$ & $80-95$ & $40-60$ & $20-50$ & 35.5 \\
\hline
\end{tabular}




\begin{tabular}{|c|c|c|c|c|c|}
\hline $\begin{array}{c}\text { Specific weight, } \\
\mathrm{g} / \mathrm{cm}^{3}\end{array}$ & $1.01-1.50$ & $1.005-1.150$ & $1.10-1.60$ & - & 1.55 \\
\hline $\begin{array}{c}\text { Organic } \\
\text { matter,\% } \\
\text { (in dry mud) }\end{array}$ & $20-98$ & $15-90$ & $1-15$ & to 0.5 & 1.07 \\
\hline $\begin{array}{c}\text { Hydrogen } \\
\text { sulfide, } \% \\
\text { (in dry mud) }\end{array}$ & - & $0.01-0.05$ & $0.05-0.50$ & 0.02 & 0.17 \\
\hline $\begin{array}{c}\text { pH-test of the } \\
\text { mud }\end{array}$ & 2.8 & $6.6-7.5$ & $7.0-9.7$ & $5.0<$ & 7.7 \\
\hline
\end{tabular}

In the Table 1 showed that peloid of this the deposit according to international classification belongs to hydrogen sulfide muddy type.

\section{Mineral and element composition in peloid from Lake Nogoon}

We determined total content of mineral composition results have shown in Table 2 and in peloid from Lake Nogoon by X-Ray Figure1. diffractometer MAXimax tool. The mineral

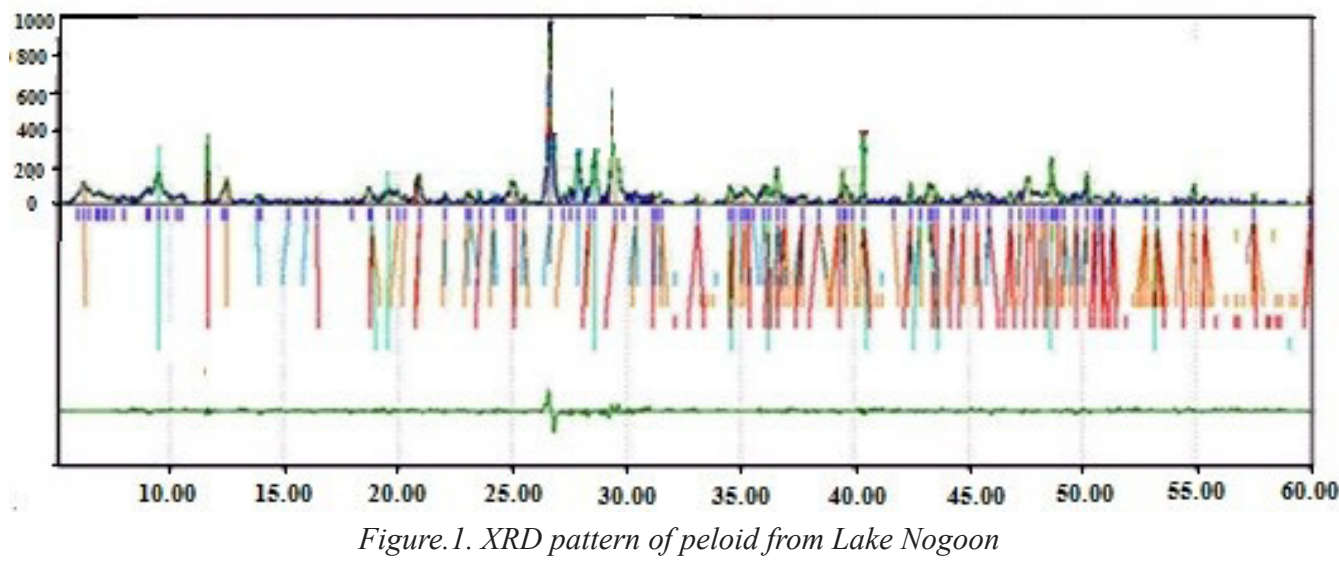

Table 2. Result of mineral composition in peloid from Lake Nogoon

\begin{tabular}{|c|c|c|c|c|}
\hline Group & \multicolumn{2}{|c|}{ Mineral name } & Mineral formula & Content, $\%$ \\
\hline \multirow{4}{*}{ Silicate } & \multirow{2}{*}{$\begin{array}{l}\text { Tecto- } \\
\text { silicates }\end{array}$} & Alibite & $\mathrm{Na}_{2} \mathrm{AlSi}_{4} \mathrm{O}_{8}$ & 13.1 \\
\hline & & Quartz & $\mathrm{SiO}_{2}$ & 22.3 \\
\hline & \multirow{2}{*}{$\begin{array}{l}\text { Phyllo- } \\
\text { silicates }\end{array}$} & Talcum & $\mathrm{Mg}_{3} \mathrm{Si}_{4} \mathrm{O}_{10}(\mathrm{OH})_{2}$ & 24.7 \\
\hline & & Clinochlore & $\left.\mathrm{Mg}_{2.96} \mathrm{Fe}_{1.55} \mathrm{Fe}_{0.136} \mathrm{Al}_{1.275}\right)\left(\mathrm{Si}_{2.622} \mathrm{Al}_{1.376}\right) \mathrm{O}_{10}(\mathrm{OH})_{8}$ & 12.2 \\
\hline Carbonate & \multicolumn{2}{|c|}{ Magnesium calcite } & $\mathrm{Mg} 0.03 \mathrm{Ca} 0.97 \mathrm{CO} 3$ & 21.8 \\
\hline Sulphate & \multicolumn{2}{|c|}{ Gypsum } & $\mathrm{CaSO} 4 \cdot 2 \mathrm{H} 2 \mathrm{O}$ & 6.0 \\
\hline
\end{tabular}

Shown this results, this peloid included the polymineral group because its contained following minerals: albite $\left(\mathrm{Na}_{2} \mathrm{AlSi}_{4} \mathrm{O}_{8}\right)$, quartz $\left(\mathrm{SiO}_{2}\right)$, talcum $\left(\mathrm{Mg}_{3} \mathrm{Si}_{4} \mathrm{O}_{10}(\mathrm{OH})_{2}\right)$, chlinochlore
$\left(\mathrm{Si}_{2.622} \mathrm{Al}_{1,376}\right) \mathrm{O}_{10}(\mathrm{OH})_{8}$, magnesium calcite $\left(\mathrm{Mg}_{0.03} \mathrm{Ca}_{0.97} \mathrm{CO}_{3}\right)$, gypsum $\left(\mathrm{CaSO}_{2} \cdot \mathrm{H}_{2} \mathrm{O}\right)$. Bedding silicate minerals developed in peloid. Thus this peloid high adsorption and ion exchange ability [9]. 
Elemental composition of peloid was performed by reontgen fluorescence waves disperse AXIOSmAX . We have identified 43 elements such us $\mathrm{Al}, \mathrm{Bi}, \mathrm{Co}, \mathrm{Cr}, \mathrm{Cs}, \mathrm{Cu}, \mathrm{Pb}, \mathrm{U}$, $\mathrm{Ba}, \mathrm{As}, \mathrm{Mo}, \mathrm{Nb}$. Results are showed in Table 3 and Table 4.

Table 3.Content of macro elements in peloid, \%

\begin{tabular}{|c|c|c|c|}
\hline Oxides & $\begin{array}{c}\text { In the peloid from Lake } \\
\text { nogoon }\end{array}$ & KC-6 $[10]$ & KC-9 [10] \\
\hline $\mathrm{SiO}_{2}$ & 43.65 & 41.62 & 33.49 \\
\hline $\mathrm{Al}_{2} \mathrm{O}_{3}$ & 7.3 & 12.82 & 6.24 \\
\hline $\mathrm{Fe}_{2} \mathrm{O}_{3}$ & 6.08 & 5.13 & 5.40 \\
\hline $\mathrm{CaO}$ & 8.7 & 13.93 & 16.70 \\
\hline $\mathrm{MgO}$ & 15.34 & 1.92 & 9.95 \\
\hline $\mathrm{Na}_{2} \mathrm{O}$ & 0.85 & 0.84 & 1.36 \\
\hline $\mathrm{K}_{2} \mathrm{O}$ & 0.71 & 2.93 & 1.03 \\
\hline $\mathrm{TiO}_{2}$ & 0.285 & 0.44 & 0.38 \\
\hline $\mathrm{MnO}^{\mathrm{P} O} \mathrm{O}_{5}$ & 0.182 & 0.06 & 0.15 \\
\hline $\mathrm{SO}_{2}$ & 0.101 & 0.19 & 0.06 \\
\hline $\mathrm{LoI}$ & 0.91 & $=$ & 24.9 \\
\hline $\mathrm{Na}_{2} \mathrm{O} / \mathrm{CaO}$ & 14.07 & 19.3 & 0.08 \\
\hline
\end{tabular}

The Table 3 shows that contents of macro elements $\mathrm{SiO}_{2}, \mathrm{MgO}$ and $\mathrm{Al}_{2} \mathrm{O}_{3}$ were $43.65 \%$, $15.34 \%$ and $7.3 \%$, respectively and this peloid is first sediment. A high $\mathrm{Na}_{2} \mathrm{O} / \mathrm{CaO}$ ratio indicates the presence of swelling 2:1 clay minerals $\left(1<\mathrm{Na}_{2} \mathrm{O} / \mathrm{CaO}>3\right)$, while a low ratio $\left(\mathrm{Na}_{2} \mathrm{O} / \mathrm{CaO}<1\right)$ is typical for non-swelling 2:1 clay minerals $[11,12]$.

$\mathrm{The} \mathrm{Na}_{2} \mathrm{O} / \mathrm{CaO}$ ratio of the mud was higher than 1.0, representing non-swelling clay minerals. And $\mathrm{Na}_{2} \mathrm{O} / \mathrm{CaO}$ ratios in this sample were found to be adove than those of Turkish peloids (KC-6 and KC-9).

Table 4. Content of elements in peloid, (\%)

\begin{tabular}{|c|c|c|c|}
\hline \multicolumn{2}{|c|}{ Elements } & $\begin{array}{c}\text { Clark elements of sea } \\
\text { bottom sediment }\end{array}$ & $\begin{array}{c}\text { In the peloid from Lake } \\
\text { nogoon }\end{array}$ \\
\hline \multirow{4}{*}{ Micro elements } & $\mathrm{Cu}$ & 0.0087 & 0.0053 \\
\cline { 2 - 4 } & $\mathrm{Cr}$ & 0.017 & 0.0998 \\
\cline { 2 - 4 } & $\mathrm{Co}$ & 0.0048 & 0.0045 \\
\cline { 2 - 4 } & $\mathrm{V}$ & 0.025 & 0.0086 \\
\cline { 2 - 4 } & $\mathrm{Zn}$ & 0.0105 & 0.0076 \\
\cline { 2 - 4 } & $\mathrm{Ni}$ & 0.013 & 0.0649 \\
\cline { 2 - 4 } & $\mathrm{Mo}$ & 0.0003 & 0.0005 \\
\hline \multirow{3}{*}{ Rare earth elements } & $\mathrm{Ce}$ & 0.007 & 0.003 \\
\cline { 2 - 4 } & $\mathrm{La}$ & 0.003 & 0.003 \\
\cline { 2 - 4 } & $\mathrm{Nd}$ & 0.0037 & 0.005 \\
\cline { 2 - 4 } & $\mathrm{Pr}$ & 0.0009 & 0.003 \\
\hline
\end{tabular}




\begin{tabular}{|c|c|c|c|}
\hline \multirow{4}{*}{ Heavy and hazardous } & $\mathrm{U}$ & 0.00026 & 0.0005 \\
\cline { 2 - 4 } & $\mathrm{W}$ & - & 0.0008 \\
\cline { 2 - 4 } & $\mathrm{Th}$ & 0.0013 & 0.0004 \\
\cline { 2 - 4 } & $\mathrm{As}$ & - & 0.0005 \\
\cline { 2 - 4 } & $\mathrm{Pb}$ & 0.025 & 0.0013 \\
\hline & $\mathrm{Bi}$ & 0.00002 & 0.0005 \\
\cline { 2 - 4 } & $\mathrm{Nb}$ & 0.0018 & 0.0003 \\
\cline { 2 - 4 } & $\mathrm{Rb}$ & 0.0078 & 0.0021 \\
\cline { 2 - 4 } & $\mathrm{Sb}$ & 0.05 & 0.004 \\
\cline { 2 - 4 } & $\mathrm{Sc}$ & 0.001 & 0.0013 \\
\cline { 2 - 4 } & $\mathrm{Sm}$ & - & 0.003 \\
\cline { 2 - 4 } & $\mathrm{Sr}$ & 0.014 & 0.0283 \\
\cline { 2 - 4 } & $\mathrm{Ta}$ & 0.00024 & 0.001 \\
\cline { 2 - 4 } & $\mathrm{Y}$ & 0.0026 & 0.0012 \\
\cline { 2 - 4 } & $\mathrm{Ga}$ & 0.0019 & 0.0009 \\
\cline { 2 - 4 } & $\mathrm{Ge}$ & 0.00015 & 0.0003 \\
\cline { 2 - 4 } & $\mathrm{Gr}$ & 0.0004 & 0.0015 \\
\cline { 2 - 4 } & $\mathrm{Cs}$ & 0.00037 & 0.0015 \\
\cline { 2 - 4 } & & & \\
\cline { 2 - 4 } & & & \\
\hline
\end{tabular}

Semi-quantitative results of Nogoon lake's peloid show that contents of some micro elements $(\mathrm{Cu} 0.0053 \%$, Co $0.0045 \%$, Zn $0.0076 \%$ ) were less than clark elements of sea bottom sediment, other micro elements (Cr $0.0998 \%$, Ni $0.0649 \%$ ) were more than clark elements of sea bottom sediment; some rare earth elements ( $\operatorname{Pr} 0.003 \%, \mathrm{Nd} 0.005 \%)$ were more than clark elements of sea bottom sediment; some heavy and hazardous elements (Th $0.0004 \%$ ) were less than clark elements of sea bottom sediment; some other elements
(Nb $0.0003 \%, \mathrm{Sb} 0.004 \%$, Y $0.0012 \%$, Ga $0.0009 \%$, Ge $0.0003 \%$ ) were less than clark elements of sea bottom sediment, other elements ( $\mathrm{Sr} 0.0283 \%$, Bi $0.0005 \%$, Hf $0.0015 \%$ ) were more than clark elements of sea bottom sediment. Several accumlation of chromium and nickel have been discovered in Govi-Altai province. Values are slightly elevated for $\mathrm{Cr}$, $\mathrm{Ni}$ concentrations in peloid from Nogoon Lake in Govi-Altai province, which is related to accumulation of chromium and nickel in the areas surrounding.

\section{Content of organic matter in peloid from Lake Nogoon}

In peloids included multiple organic matters and some biological active substances. For example; biological active substances are humic substance, lipid, and carbohydrate etc. We determined their biological activity substance's yields and which is shown on table 5.
As shown in table 5, the total organic matter yields was Lake Nogoon $1.55 \%$. In total organic matter humic acid comprises $40 \%$, lipid $24.94 \%$ and carbohydrate $0.90 \%$ respectively.

The free organic components isolated by extraction have shown in Table 6. 
Table 5. Yield of biological active substances

\begin{tabular}{|c|c|c|}
\hline \multirow{2}{*}{ Organic matter } & \multicolumn{2}{|c|}{ Total organic matter, $1.55 \%$} \\
\cline { 2 - 3 } & In dry mud, $\%$ & In total organic matter, $\%$ \\
\hline Humic substance & 0.62 & 40 \\
\hline Lipid & 0.3867 & 24.94 \\
\hline carbohydrate & 0.014 & 0.9 \\
\hline
\end{tabular}

Table 6. Free organic components of peloid

\begin{tabular}{|c|c|c|}
\hline \multirow{2}{*}{ Solvents } & \multicolumn{2}{|c|}{ Lake Nogoon } \\
\cline { 2 - 3 } & Extraction time, hour & Yield, \% (in dry mud) \\
\hline a. Hexane & 7 & 1.0891 \\
\hline b. Chloroform & 20 & 0.0805 \\
\hline c. Acetone & 42 & 0.1663 \\
\hline d. Ethanol: Benzene $/ 1: 9 /$ & 56 & 0.1438 \\
\hline Amount & 125 & 1.4797 \\
\hline
\end{tabular}

Peloid extracted effectively by hexane indicates inclusion of non-polar organic compound. On the basis of determination of total carbon, confirmed that total organic matter in peloid was more than organic substances by extraction. This suggested that organic matter in peloid contain another kind of organic compound.

We determined chloroform-dissoluble matter in total extract's dry residue after consistently extraction by organic solvents by IR and chromato-mass-spectrometric methods. Mass spectrometry to identify total
83 organic compounds in peloid from Lake Nogoon contained alkanes $<\mathrm{C}_{13} 0.56 \%, \mathrm{C}_{13}-\mathrm{C}_{19}$ $3.04 \%, \mathrm{C}_{20}-\mathrm{C}_{31}$ 8.47\%,DAF $3.09 \%$, element sulfur $39.34 \%$, cyclic hydrocarbons $0.67 \%$, halogen bearing hydrocarbons $20.8 \%$, steroids $2.32 \%$, aromatic carbohydrates $0.14 \%$, ketone $1.01 \%$, nitrogen-containing compound $3.96 \%$ and silicon-containing compound $5.06 \%$ respectively.

Chloroform-dissoluble matter in total extract's dry residue after extraction by chloroform determined by infra red (IR). The results are shown in Figure 2.

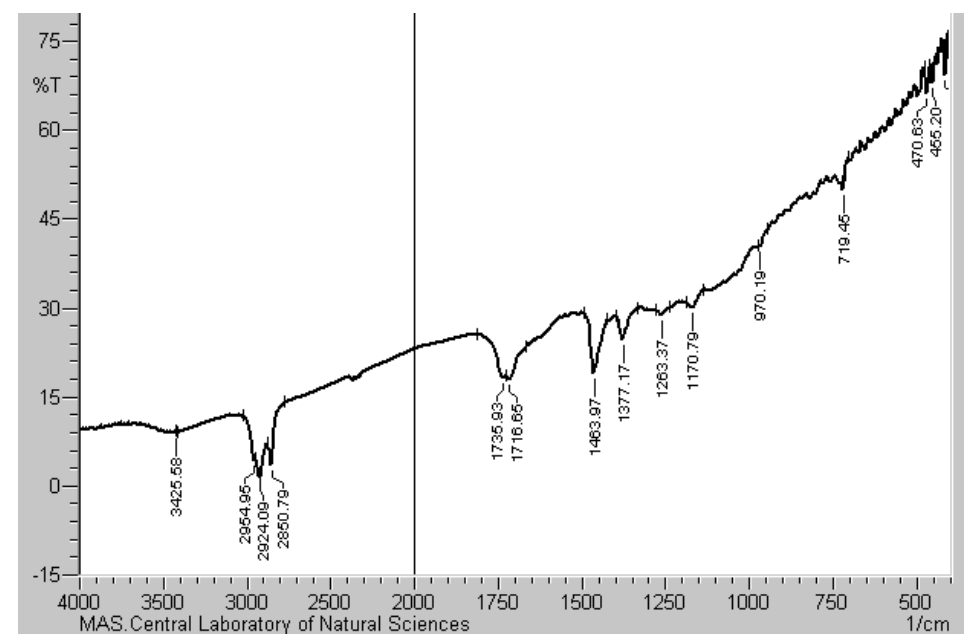

Figure. 2. IR spectrum of peloid 
The following adsorption spectrum in IR spectrum was identified in peloid from Lake Nogoon. The broad band at $3448.72 \mathrm{~cm}^{-1}$ was assigned to $\mathrm{OH}$ and $\mathrm{NH}$ groups. The intense bands at $2924.09 \mathrm{~cm}^{-1}-2654.95 \mathrm{~cm}^{-1}$ were assigned $\mathrm{CH}, \mathrm{CH} 2$, and $\mathrm{CH} 3$ aliphatic. The wide bands at $1716.6 \mathrm{~cm}^{-1}-1735.9 \mathrm{~cm}^{-1}$ were assigned $\mathrm{C}=\mathrm{C}$ valence variance and $(\mathrm{CO})$ carbonyl groups. The band at $1377.17 \mathrm{~cm}^{-1}$ was assigned $\mathrm{CH}_{3}, \mathrm{CH}_{2}$ and amines $\mathrm{C}-\mathrm{N}$ group. The band at $1263.3 \mathrm{~cm}^{-1}$ was $\mathrm{C}-\mathrm{O}$ group's valence variance and $1170.7 \mathrm{~cm}^{-1}$ was assigned alcohol, carboxylic acid, ether (C-O) group. The band at $970.1 \mathrm{~cm}^{-1}$ was $\mathrm{C}-\mathrm{H}$ deformation variance. The 719.4-719.45 $\mathrm{cm}^{-1}$ bands were assigned to carboxylic acid -C-Cl.

The lipids are a large and diverse group of naturally occurring organic compounds that are related by their solubility in nonpolar organic solvents (e.g. ether, chloroform, acetone and benzene) and general insolubility in water. The lipid fraction isolated from this peloid and extracted by mixture of ethanol: benzene $(1: 1)$.

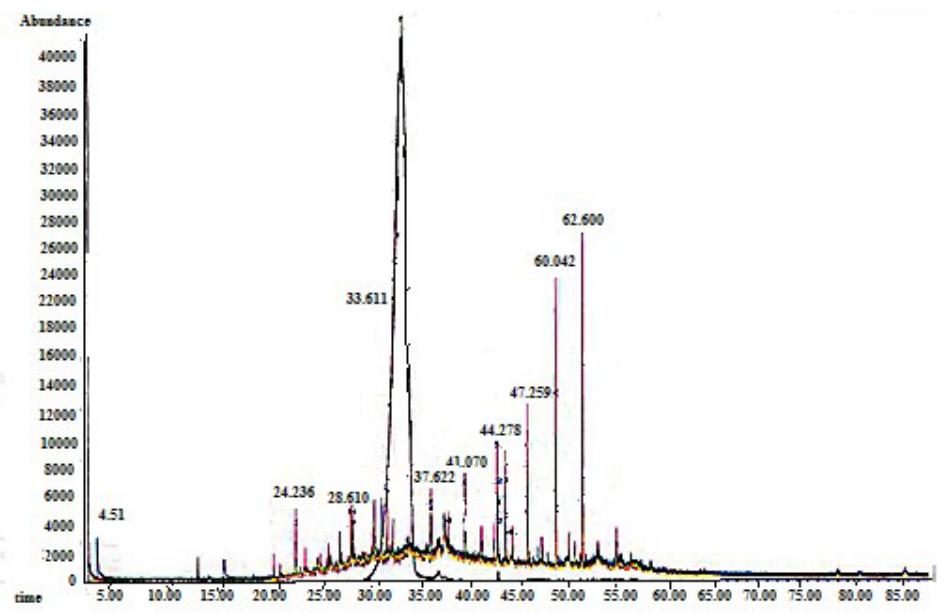

Figure.3. Chromatogram of lipid in peloid from Lake Nogoon

Table 7. Lipid matter of peloid from Lake Nogoon, \%

\begin{tabular}{|c|c|c|c|c|c|c|c|}
\hline \multirow{3}{*}{ 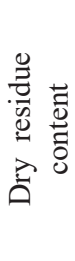 } & \multicolumn{7}{|c|}{ Organic matters in dry extract of lipid, $\%$} \\
\hline & \multicolumn{2}{|c|}{ Carbohydrates } & \multicolumn{2}{|c|}{ Carboxylic acid and their ether } & \multirow{2}{*}{ 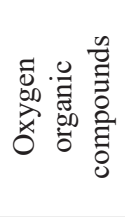 } & \multirow{2}{*}{ 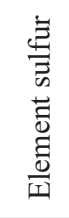 } & \multirow[b]{2}{*}{$\frac{\dot{\Phi}}{5}$} \\
\hline & $\left(\mathrm{C}_{\mathrm{n}} \mathrm{H}_{2 \mathrm{n}+2}\right)$ & $\left(\mathrm{C}_{\mathrm{n}} \mathrm{H}_{2 \mathrm{n}}\right)$ & $\begin{array}{l}\mathrm{RC}(\mathrm{O}) \mathrm{OR}^{1} \\
\left(\mathrm{R} ; \mathrm{R}^{1}\right)\end{array}$ & $\begin{array}{c}\mathrm{C}_{6} \mathrm{H}_{4}(\mathrm{COO})_{2} \mathrm{RR}^{1} \\
\left(\mathrm{R} ; \mathrm{R}^{1}\right)\end{array}$ & & & \\
\hline 0.39 & $\begin{array}{c}1.15 \\
\left(\mathrm{C}_{11}\right) \\
15.91 \\
\left(\mathrm{C}_{14}-\mathrm{C}_{18}\right) \\
23.48 \\
\left(\mathrm{C}_{20}-\mathrm{C}_{24}\right) \\
7.19 \\
\left(\mathrm{C}_{25}-\mathrm{C} 29\right) \\
47.73\end{array}$ & - & $\begin{array}{c}0.4 \\
\left(\mathrm{C}_{17} \mathrm{H}_{33} ; \mathrm{H}\right) \\
0,3 \\
\left(\mathrm{C}_{17} \mathrm{H}_{35} ;\right. \\
\left.\mathrm{C}_{4} \mathrm{H}_{9}\right) \\
0.7\end{array}$ & $\begin{array}{c}5.58 \\
\left(\mathrm{C}_{4} \mathrm{H}_{9} ; \mathrm{C}_{4} \mathrm{H}_{9}\right) \\
5.58\end{array}$ & $\begin{array}{l}0.17 \\
0.43 \\
\\
0.6\end{array}$ & 2.88 & $\begin{array}{c}0.27^{1} \\
17.24^{2} \\
2.33^{3} \\
3.47^{4} \\
0.15\end{array}$ \\
\hline
\end{tabular}

Note: ${ }^{1}$ - cyclic hydrocarbons, ${ }^{2}-$ Steroids, ${ }^{3}$ - nitrogen-containing compound, ${ }^{4}-$ silicon-containing compound. 
From Table 7 shows that in peloid from Lake Nogoon lipid matter contained 55 organic compounds alkanes $47.73 \%$, DAF $5.58 \%$, carboxylic acid $0.7 \%$, ester $0.3 \%$, steroids $17.24 \%$, oxygen organic compound $0.6 \%$, and

\section{CONCLUSION}

1. We have studied general properties and chemical composition of peloid from Nogoon Lake. Result of this analysis show Nogoon Lake's mud according to international classification belongs to silt sulphide muddy type.

2. The IR spectrum of peloid from Lake Nogoon showed $\mathrm{CH}_{2}, \mathrm{CH}_{3}$ alkanes group, carboxylic acid, ether (C-O) group, alkanes $(\mathrm{CH})$ groups absorption that free organic matter in peloid consist multiple compounds.

3. The GS/MS chromatogram of dissoluble organic matter (DOM) from Lake Nogoon contained hydrocarbons and their derivatives of $33.68 \%$, dialkylphatalate element sulfur $2.88 \%$, nitrogen-containing compound $\quad 2.33 \%, \quad$ silicon-containing compound $3.74 \%$, cyclic hydrocarbons $0.27 \%$ respectively.

(DAF) of $3.09 \%$, element sulfur $39.34 \%$, cyclic hydrocarbons $0.67 \%$, halogen bearing hydrocarbons $20.8 \%$, steroids $2.32 \%$, aromatic carbohydrates $0.14 \%$, ketone $1.01 \%$, nitrogencontaining compound $3.96 \%$ and siliconcontaining compound $5.06 \%$ respectively.

4. It was estimated that in peloid from Lake Nogoon lipid matter contained 55 organic compounds alkanes $47.73 \%$, DAF $5.58 \%$, carboxylic acid $0.7 \%$, ester $0.3 \%$, steroids $17.24 \%$, oxygen organic compound $0.6 \%$, and element sulfur $2.88 \%$, nitrogen-containing compound $\quad 2.33 \%, \quad$ silicon-containing compound $3.74 \%$, cyclic hydrocarbons $0.27 \%$ respectively. 


\section{REFERENCES}

1. Dolmaa, G., Ugtakhbayar, O., Dubinskaya, E.I., Tserenpil, Sh., Voronkov, M.G., Lipids in medicinal mud of some lakes Mongolia // the second Intern. Conf.on Chemical Investigation and Utilization of natural Recources. p. 111, Ulaanbaatar, 2003.

2. Dolmaa, G., Peloid is magnificent product of nature, p. 9-10, Ulaanbaatar, (2012).

3. Tsogtgerel, D Chemical investigation of some peloid from Gobi-Altai province, p.18, Ulaanbaatar, (2013).

4. $\quad$ MNS 5848-2008. Medical mud classification. Technical requirements

5. MNS 5849:2008. Determination of chemical, physical method for medical mud

6. MNS 5443-2005. Method of determination total lipid contents in medical mud

7. MNS 5442-2005. Method of determination of humic compounds content in medical mud

8. $\quad$ Types of medical mud. www.doctor-limus.ru/en/types.html"

9. Nelson Eby, G., Principles of environmental Geochemisrty. Brooks/Cole-Thomson Learning, p. 219-233, (2004)

10. Muazzez Çelik Karakaya., Necati Karakaya., Şerife Sarıoğlan., Murat Koral., Some properties of thermal muds of some spas in Turkey. Applied Clay Science 48, p.531-537, (2010).

11. Ravaglioli, A., Fiori, C., Fabbri, B., In: Faenza (Ed.) Materia Prime Ceramiche. Argille, materiali non argillosi e sottoprodottiindustriali. BibliotecaTecnica Ceramica, p.392 (1989).

12. Cara, S., Carcangiu, G., Paladino, G., Palomba, M., Tamanini, M., the bentonites in pelotherapy: thermal properties of clay pastes from Sardinian (Italy). Applied ClayScience 16, p.125-132, Italy (2000) 